\title{
Antioxidative fullerol promotes osteogenesis of human adipose-derived stem cells
}

This article was published in the following Dove Press journal: International Journal of Nanomedicine

20 August 2014

Number of times this article has been viewed

\section{Xinlin Yang \\ Ching-Ju Li \\ Yueping Wan \\ Pinar Smith \\ Guowei Shang \\ Quanjun Cui}

Department of Orthopaedic Surgery, University of Virginia School of Medicine, Charlottesville, VA, USA
Correspondence: Quanjun Cui Department of Orthopaedic Surgery University of Virginia School of Medicine Hospital Drive, Cobb Hall B053, PO Box 800374 Charlottesville, VA 22903, USA

Tel +l 4342430266

Fax +I 4342430242

Email qc4q@hscmail.mcc.virginia.edu
Abstract: Antioxidants were implicated as potential reagents to enhance osteogenesis, and nano-fullerenes have been demonstrated to have a great antioxidative capacity by both in vitro and in vivo experiments. In this study, we assessed the impact of a polyhydroxylated fullerene, fullerol, on the osteogenic differentiation of human adipose-derived stem cells (ADSCs). Fullerol was not toxic against human ADSCs at concentrations up to $10 \mu \mathrm{M}$. At a concentration of $1 \mu \mathrm{M}$, fullerol reduced cellular reactive oxygen species after a 5-day incubation either in the presence or in the absence of osteogenic media. Pretreatment of fullerol for 7 days increased the osteogenic potential of human ADSCs. Furthermore, when incubated together with osteogenic medium, fullerol promoted osteogenic differentiation in a dose-dependent manner. Finally, fullerol proved to promote expression of FoxO1, a major functional isoform of forkhead box $\mathrm{O}$ transcription factors that defend against reactive oxygen species in bone. Although further clarification of related mechanisms is required, the findings may help further development of a novel approach for bone repair, using combined treatment of nano-fullerol with ADSCs.

Keywords: polyhydroxylated fullerene, bone repair, reactive oxygen species, forkhead box protein $\mathrm{O} 1$

\section{Introduction}

Traumatic injuries and pathological conditions, such as osteoporosis, osteonecrosis, and bone tumors, can lead to bone fractures that do not heal through endogenous mechanisms. These difficult fractures in individuals are a serious threat to the health of the workforce and to the economy. In 2008, a survey showed that 110 million adults in the US reported disabling musculoskeletal conditions, and a World Health Organization report showed that $50 \%$ of the 9 million osteoporotic fractures that had occurred worldwide had happened in the Americas and Europe. ${ }^{1,2}$ It is estimated that of the 7.9 million fractures sustained each year in the United States, $5 \%$ to $20 \%$ result in delayed or impaired healing. ${ }^{2}$ Therefore, there is a need for effective, low cost, and innovative approaches of bone repair for musculoskeletal injuries and disease.

Application of stem cells represents one of the most valued advances in bone tissue engineering, which offers promise for bone repair. ${ }^{3}$ Mesenchymal stem cells (MSCs) have the ability to differentiate along various lineages of mesenchymal origin, including chondrocyte, osteoblast, and adipocyte lineages, depending upon the biological environment. Moreover, MSCs can be obtained from multiple adult tissues, such as bone marrow, trabecular bone, articular cartilage, muscle, and adipose. ${ }^{4}$ Retrieval of adipose tissue involves a minimally invasive procedure that can be easily performed in outpatient clinics, and the yield of adipose-derived stem cells (ADSCs) is about $5 \times 10^{3}$ cells per gram of tissue, which is 100 -fold higher than the number of stem cells from bone marrow. ${ }^{5}$ Therefore, adipose tissue may be a better source of stem cells for potential clinical use. Although the efficacy of ADSCs to enhance bone repair 
has not yet been investigated in clinical trials, ADSCs have been reported to enhance bone healing in various animal models. ${ }^{6-11}$

While combined delivery of ADSCs and growth factors has been reported to enhance osteogenesis in vivo, $, 10,12$ contradictory reports have been published that raise a serious concern about the efficacy of this approach. For example, ADSCs do not respond to various bone morphogenetic proteins (BMPs) in vitro. ${ }^{13,14}$ In addition, a recent report demonstrated that delivery of human ADSCs and BMP-2 had no advantage over delivery of BMP-2 alone to enhance fracture healing in nude rats. ${ }^{15}$ The obvious limitations of growth factors due to their short half-life and high production cost should be considered as well. Therefore, alternatives to growth factors are needed to enhance the osteogenesis of ADSCs.

Recently Yamada et $\mathrm{al}^{16}$ demonstrated by in vitro and in vivo experiments that $\mathrm{N}$-acetylcysteine, an antioxidative small molecule, could enhance the osteogenesis of rat bone marrow stromal cell and bone regeneration. We hypothesize that an antioxidant would favor osteogenic differentiation in ADSCs as well. Here, we investigated an extremely powerful antioxidant, polyhydroxylated fullerene (fullerol), ${ }^{17-19}$ and report for the first time that antioxidative fullerol enhanced osteogenesis and reduced reactive oxygen species (ROS) in human ADSCs, as well as increased expression of FoxO1, a major isoform of forkhead box $\mathrm{O}$ transcription factors that defend against ROS and promote osteoblast differentiation in bone..$^{20,21}$

\section{Materials and Methods Cell culture}

Human ADSCs were purchased from a commercial source (Lonza, Basel, Switzerland) and were characterized for osteogenic assays in our previous paper. ${ }^{22}$ The cells were maintained in basal medium that was Dulbecco's Modified Eagle's Medium (Gibco BRL, Gaithersburg, MD, USA) containing $10 \%$ fetal bovine serum (Hyclone Laboratories, Logan, UT, USA), $50 \mathrm{mg} / \mathrm{mL}$ ascorbic acid (AA), $100 \mathrm{IU} / \mathrm{mL}$ penicillin $\mathrm{G}$, and $100 \mathrm{mg} / \mathrm{mL}$ streptomycin in a humidified atmosphere of $5 \%$ carbon dioxide at $37^{\circ} \mathrm{C}$. To induce osteogenic differentiation, $10 \mathrm{mM} \beta$-glycerophosphate (GP) and $10^{-7} \mathrm{M}$ dexamethasone (DEX) were supplemented to basal medium, and the medium was designated as osteogenic medium. Fullerol (M.E.R. Co., Tucson, AZ, USA) was added when cells became 70\%-80\% confluent before (pretreatment) or at the time (cotreatment) of induction. All experiments were conducted with cells at passage 8 and a starting cell density of 5,000 cells $/ \mathrm{cm}^{2}$.
Lactate dehydrogenase (LDH) and 3-(4,5-dimethylthiazol-2-yl)-5(3-carboxymethoxyphenyl)-2-(4sulfophenyl)-2H-tetrazolium (MTS) assays Both LDH (Biovision Inc., Milpitas, CA, USA) and MTS (Promega Corporation, Fitchburg, WI, USA) colorimetric kits were used for cell toxicity and proliferative assays, respectively, by following the enclosed instructions in each product. For the LDH assay, cytotoxicity of $100 \%$ was set to the positive control where cells were treated by $1 \%$ Triton $\mathrm{X}-100$, a detergent leading to a complete release of the LDH enzyme from the cells. In the MTS assay, cells produced a colorful substance, in which the optical density value reflecting the cell number was measured at $490 \mathrm{~nm}$. Cells were treated with different doses of fullerol $(0-10 \mu \mathrm{M})$ for 6 hours and 48 hours, for LDH and MTS assays, respectively.

\section{ROS detection}

The reagent $\mathrm{CM}-\mathrm{H}_{2} \mathrm{DCF}-\mathrm{DA}$ (5-[and-6]-chloromethyl-2', $7^{\prime}$-dichlorodihydrofluorescein diacetate, acetyl ester) is a derivative of 2,7-dichlorodihydrofluorescein diacetate (DCF-DA), but with an additional thiol reactive chloromethyl group, which helps the transportation of the compound through cell membrane. The acetyl group is removed by intracellular esterase, producing DCF-DA, which can be retained within cells. The dye DCF-DA itself is nonfluorescent until cellular oxidation, at which point it becomes fluorescent. In the present experiments, ROS detection was performed by a commercial kit for DCF-DA assay (Invitrogen, Eugene, OR, USA), according to the manufacturer's manual. Briefly, after treatment, cells were rinsed with Hank's Balanced Salt Solution twice and stained with $20 \mu \mathrm{M}$ DCF-DA in Hank's Balanced Salt Solution for 30 minutes at $37^{\circ} \mathrm{C}$, and the relative fluorescence units were read on a fluorescence plate reader with an excitation wavelength of $488 \mathrm{~nm}$ and an emission wavelength of $515 \mathrm{~nm}$. The signal was then normalized by the deoxyribonucleic acid (DNA) content measured by a fluorescent dye ethidium homodimer-1 with an excitation wavelength of $528 \mathrm{~nm}$ and an emission wavelength of $617 \mathrm{~nm}$.

\section{Alizarin red staining}

Mineralization was quantified by alizarin red staining using a standardized procedure in our laboratory. ${ }^{22}$ Briefly, the cells were stained with $40 \mathrm{mM}$ alizarin red $(\mathrm{pH}=4.0)$ for 20 minutes, while the excess dye was washed away with water. After the picture of the whole plate was taken by a common digital camera, the dye bound to the deposited minerals in 
each well was extracted by $10 \%$ (volume/volume [v/v]) acetic acid. The resultant solution was then neutralized by $10 \%(\mathrm{v} / \mathrm{v})$ $\mathrm{NH}_{4} \mathrm{OH}$ and transferred to a 96 -well plate. The optical density at $405 \mathrm{~nm}$ was measured on a microplate reader.

\section{Quantitative real-time reverse transcription-polymerase chain reaction (RT-PCR)}

Total ribonucleic acid (RNA) was isolated from cells using the RNeasy ${ }^{\circledR}$ Mini kit (Qiagen, Valencia, CA, USA), and complementary DNA (cDNA) was synthesized with the Reverse Transcription System kit (Promega, Madison, WI, USA) following the manufacturers' instructions. Real-time PCR was performed with the QuantiTect ${ }^{\circledR}$ SYBR Green PCR master mix (Qiagen, Valencia, CA, USA). The threshold cycle value was calculated from amplification plots. Data were analyzed using the $2^{-\triangle \Delta \mathrm{CT}}$ method with $18 \mathrm{~s}$ ribosomal RNA (rRNA) serving as the reference. ${ }^{23}$ Gene expression was normalized to the control group in each experiment and represented as fold of change. The target genes including runt-related transcription factor 2 (Runx2), osteocalcin (OCN), alkaline phosphatase (ALP), peroxisome proliferator-activated receptor gamma (PPARg), and leptin (LEP), as well as their primer sequences and product sizes are shown in Table 1.

\section{Western blots}

The cells (approximately 500,000) from one well of a 6-well plate were lysed with sodium dodecyl sulfate (SDS) sample buffer, without bromophenol blue and consisting of $125 \mathrm{mM}$ Tris- $\mathrm{HCl}$ pH 6.8, 150 mM $\beta$-mercaptoethanol, 1\% SDS, 20\% glycerol, $1 \mathrm{X}$ protease inhibitor cocktail (Santa Cruz Biotechnology Inc., Dallas, TX, USA) and $1 \mathrm{mM}$ phenylmethylsulfonyl fluoride (Santa Cruz Biotechnology Inc.). The lysates were homogenized by ultrasonic homogenizer and centrifuged at 20,000 rpm for 20 minutes at $4^{\circ} \mathrm{C}$ to remove cell debris. Clear supernatants were transferred to a precooled fresh tube and immediately placed on ice. The protein concentration of the cell lysates was determined using a Bradford protein assay kit (Bio-Rad, Hercules, CA, USA). One hundred $\mu \mathrm{g}$ of total proteins were run on 5\%-12\% SDS-polyacrylamide gels at constant current of $80 \mathrm{~V}$ and electro-transferred to nitrocellulose membranes (Thermo Scientific, Waltham, MA, USA) at constant voltage of $10 \mathrm{~V}$ overnight. Membranes were blocked with $5 \%$ bovine serum albumin in Tris buffered saline with Tween solution (50 mM Tris, $\mathrm{pH} 7.6,150 \mathrm{mM} \mathrm{NaCl}$, $0.05 \%$ Tween 20 ) for 1 hour at room temperature, washed, and incubated overnight at $4^{\circ} \mathrm{C}$ in $5 \%$ bovine serum albumin in Tris buffered saline with Tween solution containing specific antibodies including anti-OCN(Santa Cruz Biotechnology Inc.), anti-Runx2 (Cell Signaling, Danvers, MA, USA), anti-superoxide dismutase (SOD) 2 (Santa Cruz Biotechnology Inc.), anti-FoxO1a (Abcam, Cambridge, MA, USA), anti- $\beta$-actin (Sigma-Aldrich Co., St Louis, MO, USA) and anti-glyceraldehyde 3-phosphate dehydrogenase (GAPDH) (Cell Signaling). The membranes were then incubated with horseradish peroxidase-conjugated secondary antibody (Cell Signaling) for 1 hour at room temperature followed by chemiluminescent substrate for horseradish peroxidase antibody and enhancer solution (Thermo Scientific) mixed in a 1:1 ratio. The membranes were incubated in the dark with autoradiography films (Genesee Scientific, San Diego, CA, USA), and the films were developed to visualize the bands. Quantifying band density: The grayscale images (300-400 dpi) on films were scanned using a flatbed scanner. Densitometry was carried out in Photoshop (Adobe Systems, Inc., San Jose, CA, USA). Using the magic wand tool from the tool palette, the area of each band was selected, and the mean histogram was recorded and then charted. All Western blots were conducted in duplicates.

Table I Target genes, primer sequences, and product sizes for quantitative real-time polymerase chain reaction

\begin{tabular}{|c|c|c|}
\hline Molecule & Primer sequences & Size of product (bp) \\
\hline Runt-related transcription factor & 5'-AGATGATGACACTGCCACCTCTG-3' (sense) & 125 \\
\hline 2 (Runx2) & 5'-GGGATGAAATGCTTGGGAACTGC-3' (antisense) & \\
\hline \multirow[t]{2}{*}{ Alkaline phosphatase (ALP) } & 5'-ACCATTCCCACGTCTTCACATTTG-3' (sense) & 162 \\
\hline & 5'-AGACATTCTCTCGTTCACCGCC-3' (antisense) & \\
\hline \multirow[t]{2}{*}{ Osteocalcin (OCN) } & 5'-CAGCGAGGTAGTGAAGAGAC-3' (sense) & 144 \\
\hline & 5'-TGAAAGCCGATGTGGTCAG-3' (antisense) & \\
\hline Peroxisome proliferator-activated & 5'-CCTGAAACTTCAAGAGTACCA-3' (sense) & 96 \\
\hline receptor gamma (PPARg) & 5'-TCATGAGGCTTATTGTAGAGC-3' (antisense) & \\
\hline \multirow[t]{2}{*}{ Leptin (LEP) } & 5'-GTGCGGATTCTTGTGGCTTT-3' (sense) & 174 \\
\hline & 5'-GGAATGAAGTCCAAACCGGTG-3' (antisense) & \\
\hline \multirow[t]{2}{*}{$18 \mathrm{~s}$} & 5'-GTGACCAGTTCACTCTTGGT-3' (antisense) & 99 \\
\hline & 5'-GAATCGAACCCTGATTCCCCGTC-3' (antisense) & \\
\hline
\end{tabular}

Abbreviation: bp, base pair. 


\section{Data analysis}

Data from the analyses of ROS, mineralization and gene expression were expressed as mean $\tilde{\mathrm{A}} \pm$ standard deviation (SD). Statistical evaluation was performed with two-tailed Student's $t$-test using Microsoft Excel ${ }^{\circledR}$ (Microsoft Corporation, Redmond, WA, USA) to determine the difference between two groups, with a $P$-value of 0.05 or less considered as a significant difference.

\section{Results}

\section{Fullerol displays no toxicity against human ADSCs}

Figure 1A displayed the results from the assay of cytotoxicity in human ADSCs, represented by LDH release from cells. Cytotoxic activities of control and fullerol groups of various doses $(0.1,0.3,1,3$, and $10 \mu \mathrm{M})$ for 6 hours were all less than $10 \%$, and no significant difference was found between the control and any fullerol group. Figure 1B was obtained from the proliferative assay for cells treated with the same set of fullerol doses for 48 hours. Again, there were no significant changes in the cell number between the control and any fullerol group.

\section{Fullerol decreases intracellular ROS level during osteogenic induction of human ADSCs}

Fullerol at two doses (low $=0.1 \mu \mathrm{M}$ and high $=1 \mu \mathrm{M}$ ) was further tested for the ROS scavenging activity in human ADSCs during a 5-day culture. The results are shown in Figure 2. With basal medium, treatment of both high and low doses significantly reduced the intracellular ROS level. The ROS levels in all concentrations of osteogenic medium were significantly lower than that in the basal medium

A

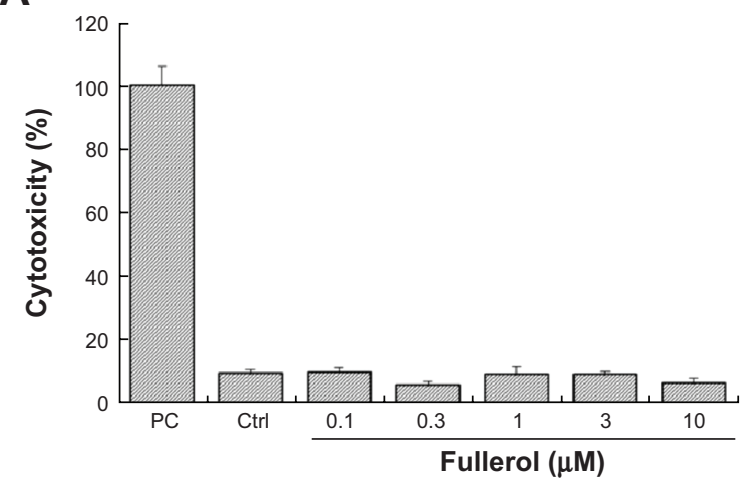

control group. Furthermore, in the presence of osteogenic medium, the high dose group, but not the low dose group, had an ROS level that was significantly lower than the control group.

\section{Pretreatment with fullerol increases osteogenic potential of human ADSCs}

We then investigated whether fullerol pretreatment was able to enhance the osteogenic potential of human ADSCs while it reduced intracellular ROS level. Osteogenesis was assessed by analyses of both the mineralization and gene expression of osteogenic markers. Quantitative RT-PCR analysis showed that cells treated with an osteogenic medium lacking AA (ie, basal medium containing GP and DEX) and fullerol for 7 days had a significantly higher expression of Runx2,OCN, and ALP compared with that of cells treated with the AA-free osteogenic medium alone (Figure 3A). Importantly, after incubation in a complete osteogenic medium for a further 2 weeks, cells with fullerol pretreatment exhibited much more mineralization by alizarin red staining (Figure 3B).

\section{Fullerol promotes osteogenic differentiation of human ADSCs}

The effects of fullerol on osteogenic differentiation of human ADSCs were determined independently as well as in combination with osteogenic medium. Treatment of fullerol at the high dose $(1 \mu \mathrm{M})$ for 13 days was determined via alizarin red staining to significantly enhance the mineralization (Figure 4A and 4B). Accordingly, expression of osteogenic markers was significantly elevated in the presence of high dose fullerol by quantitative RT-PCR analysis ( $A L P$ and $O C N$, Figure $4 \mathrm{C}$ ) and Western blot analysis (OCN, Figure 4D). Interestingly,

B

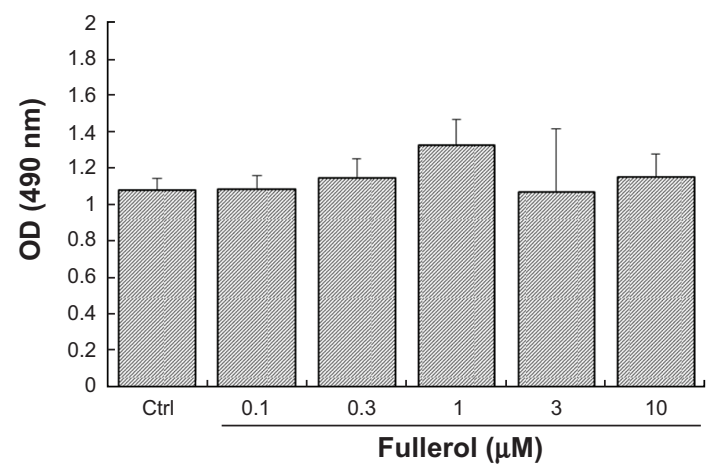

Figure I Assays for cell survival and cell proliferation in human ADSCs treated with different doses of fullerol $(I 0,3, I, 0.3,0.1$, and $0 \mu M)(n=4)$. Notes: (A) LDH assays with 6-hour treatment of fullerol. (B) MTS assays with 48-hour treatment of fullerol.

Abbreviations: ADSCs, adipose-derived stem cells; Ctrl, control group without any treatment; LDH, lactate dehydrogenase; MTS, 3-(4,5-dimethylthiazol-2-yl)5-(3-carboxymethoxyphenyl)-2-(4-sulfophenyl)-2H-tetrazolium; OD, optical density; PC, positive group for LDH assay with treatment of cells by I\% Triton X-I00 to release the highest intracellular LDH into culture medium. 


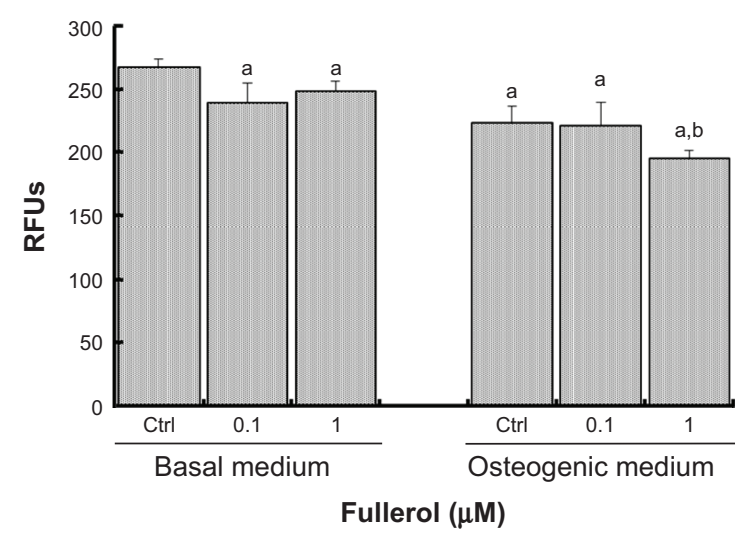

Figure 2 Fullerol reduced ROS production in human ADSCs cultured in basal medium or osteogenic medium in the presence or in the absence of fullerol $(0.1$ and I $\mu$ M) for 5 days $(n=5)$.

Notes: Intracellular ROS were monitored by a fluorescent dye DCF-DA, and the RFUs were recorded on a fluorescence plate reader. Letter a denotes $P<0.05$ versus $C$ trl in basal medium. Letter $b$ denotes $P<0.05$ versus $C$ trl in osteogenic medium. Abbreviations: ADSCs, adipose-derived stem cells; Ctrl, control; DCF-DA, 2,7dichlorodihydrofluorescein diacetate; RFUs, relative fluorescence units; ROS, reactive oxygen species.

fullerol simultaneously inhibited gene expression of adipogenic markers PPARg and LEP (Figure 4C).

\section{Fullerol augments expression of FoxOl gene expression in human ADSCs}

In an attempt to study the possible mechanistic pathway underlying promotion of osteogenesis by fullerol in human ADSCs, we investigated expression of an important transcription factor FoxO1 and its target genes Runx2 and SOD2 by Western blot analysis. FoxO1 level was relatively low in basal medium and robustly elevated after osteogenic induction. Fullerol $(1 \mu \mathrm{M})$ caused an additive increase in FoxO1 in the presence of osteogenic medium (Figure 5A). In line with its stimulation of FoxO1 expression, fullerol also augmented expression of Runx2 and SOD2 (Figure 5B).

\section{Discussion}

In the current study, we investigated the effects of antioxidative nano-fullerol on the osteogenic capacity of primary cultures of human ADSCs by conventional experimental techniques for cell biology and biochemistry, including cell survival and proliferative assays, intracellular ROS measurement, calcium deposit detection, real-time RT-PCR, and Western blotting. The outcomes are encouraging in the following areas: (1) fullerol was nontoxic toward human ADSCs at doses up to $10 \mu \mathrm{M},(2)$ fullerol was an ROS scavenger for human ADSCs, (3) pretreatment or cotreatment of fullerol enhanced osteogenic capacity of human ADSCs in culture, and (4) fullerol stimulated gene expression of FoxO1 and its target genes Runx2 and SOD2.

Fullerene has a unique structure, composed of 60 carbon atoms that form a hollow sphere about 1 nanometer in diameter. ${ }^{24}$ Capable of being reversibly reduced by up to six electrons and with as many as 34 methyl radicals added to a $\mathrm{C}_{60}$ sphere, fullerenes (fullerene $\mathrm{C}_{60}$ and its derivatives) have been characterized as "radical sponges" and are reported to have antioxidant activity several hundred-fold higher than other antioxidants. ${ }^{25}$ This was supported by our previous findings that fullerol was more powerful than a conventional antioxidant, glutathione, in inhibiting expression of adipogenic markers and stimulating expression of osteogenic markers in a mouse bone marrow stromal cell line, D1 cells. ${ }^{26} \mathrm{~A}$ variety of different fullerenes have been studied for their biomedicinal applications, and they have taken on an important role in the research areas of both nanobiotechnology and nanomedicine. ${ }^{27}$ Furthermore, due to its simple, straightforward preparation and its robust
A

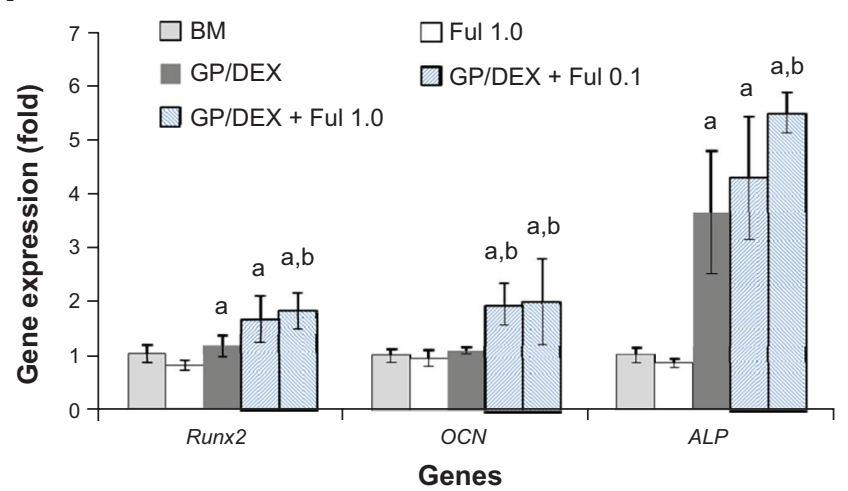

B

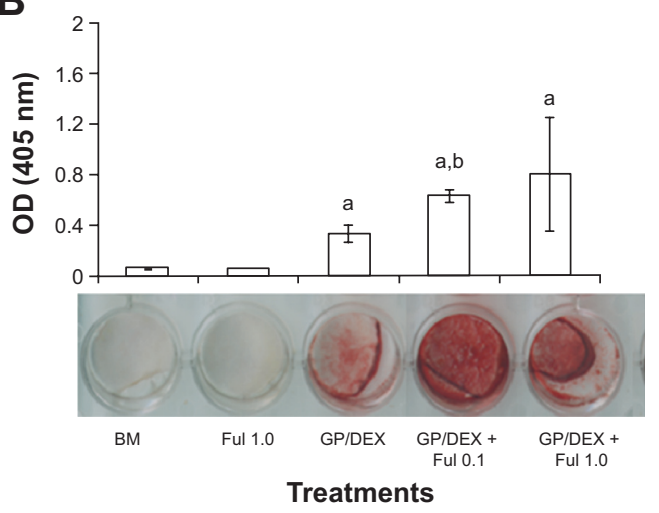

Figure 3 Fullerol pretreatment increased osteogenic potential of human ADSCs.

Notes: Cells were pretreated with fullerol (I.0 $\mu \mathrm{M}$ or $0.1 \mu \mathrm{M})$ for 7 days, followed by osteogenic induction for 14 days $(n=4)$. (A) Gene expression of osteogenic markers, Runx2, OCN, and ALP by real-time RT-PCR at day 7, using 18 s as internal control. (B) Alizarin red staining at day 2 I. Letters a and b denote $P<0.05$ versus BM and GP/DEX group, respectively.

Abbreviations: ADSCs, adipose-derived stem cells; ALP, alkaline phosphatase; BM, basal medium; Ful, fullerol; GP/DEX, glycerophosphate/dexamethasone; OCN, osteocalcin; OD, optical density; Runx2, runt-related transcription factor 2. 
A

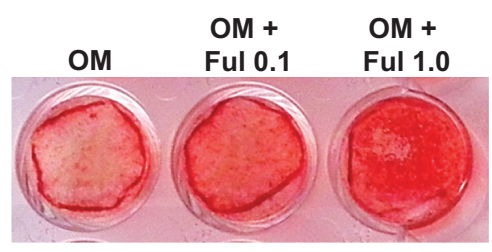

C

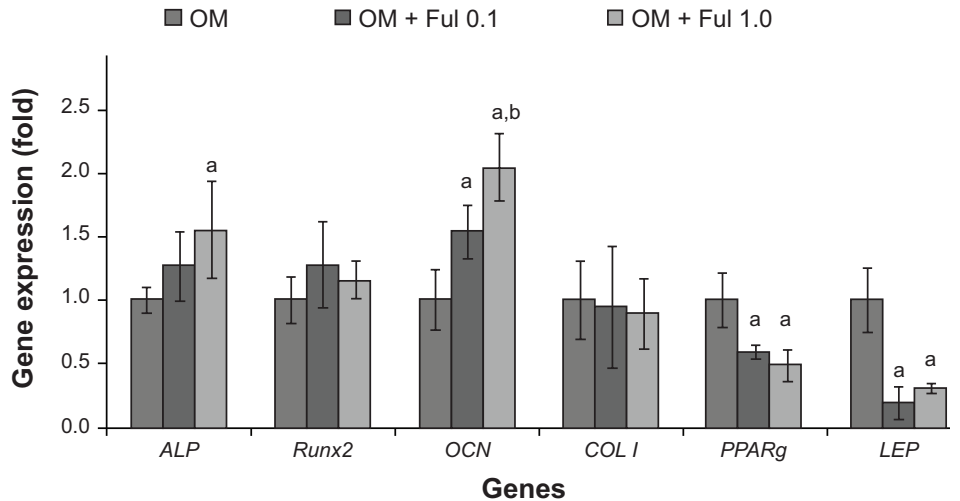

B

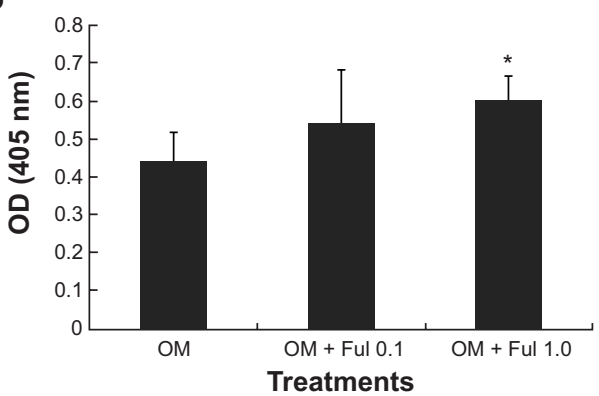

D
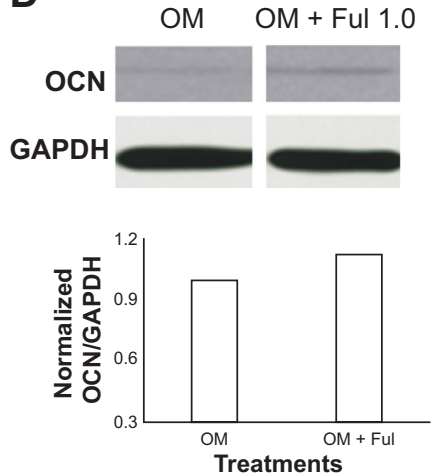

Figure 4 Osteogenic differentiation in human ADSCs treated with different doses of fullerol $(0,0.1$, and I.0 $\mu M)$ for I 3 days ( $=4)$.

Notes: (A and B) Alizarin red staining showed that fullerol increased mineralization (A: images and B: quantification); (C) Quantitative real-time RT-PCR revealed that fullerol stimulated expression of osteogenic genes ALP and OCN and inhibited expression of adipogenic genes PPARg and LEP. Letters a and $b$ denote $P<0.05$ versus $O M$ and OM + Ful 0.I group, respectively. (D) Western blots demonstrated that fullerol elevated cellular OCN protein level.

Abbreviations: ADSCs, adipose-derived stem cells; ALP, alkaline phosphatase; BM, basal medium; COL I, type I collagen; Ful, fullerol; GAPDH: glyceraldehyde 3-phosphate dehydrogenase; GP/DEX, glycerophosphate/dexamethasone; LEP, leptin; OCN, osteocalcin; OD, optical density; OM, osteogenic medium; PPARg, peroxisome proliferator-activated receptor gamma; RT-PCR, reverse transcription-polymerase chain reaction; Runx2, runt-related transcription factor 2.

A
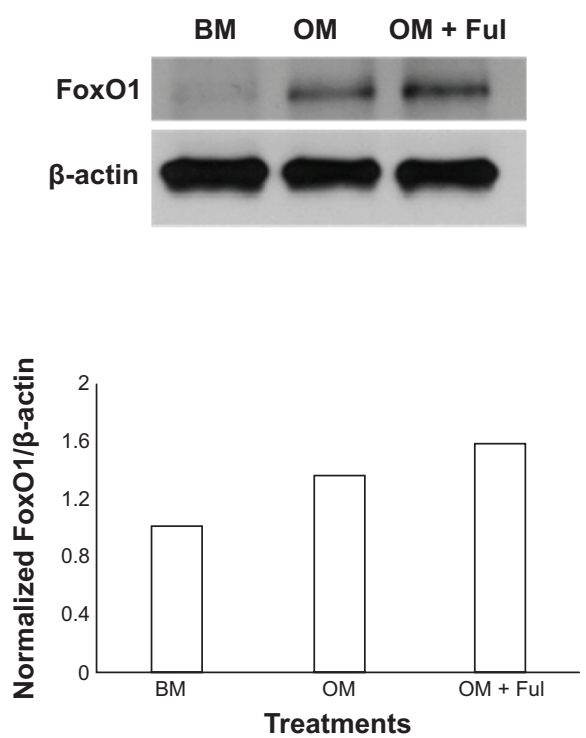

B

\section{OM SODOM + Ful}
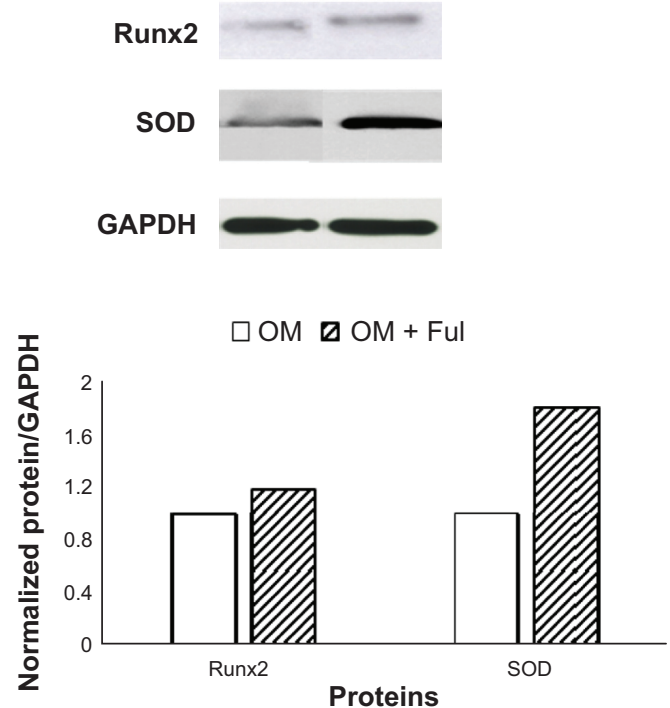

Figure 5 Fullerol promoted gene expression of FoxOI.

Notes: This transcription factor's targets, Runx2 and SOD2, were analyzed by Western blots. Human ADSCs were treated with OM alone or together with I $\mu$ M fullerol (OM + Ful) for 7 (A) or 13 (B) days.

Abbreviations: ADSCs, adipose-derived stem cells; BM, basal medium; FoxOI, forkhead box protein OI; Ful, fullerol; GAPDH, glyceraldehyde 3-phosphate dehydrogenase; $\mathrm{OD}$, optical density; OM, osteogenic medium; Runx2, runt-related transcription factor 2; SOD: superoxide dismutase. 
water solubility, ${ }^{19,28-30}$ fullerol has been considered as a candidate with priority for the biomedical purpose of fullerenes. ${ }^{31}$ Our present data showed that fullerol served as an antioxidant in human ADSCs without cytotoxicity at doses up to $10 \mu \mathrm{M}$ (Figures 1 and 2), and this is in agreement with a majority of previous reports with regards to other tissues and cell types. ${ }^{17-19,28,32-39}$

It has been shown that ROS inhibit osteogenesis and bone formation. Mody et $\mathrm{al}^{40}$ reported that three oxidants, hydrogen peroxide $\left(\mathrm{H}_{2} \mathrm{O}_{2}\right)$, xanthine, and xanthine oxidase, and minimally oxidized low-density lipoproteins inhibited osteoblastic differentiation of MC3T3-E1 mouse bone preosteoblast cell line and M2-10B4 mouse marrow stromal cell line, while increasing intracellular oxidative stress. Chen et $\mathrm{al}^{41}$ observed a dramatic decrease of ROS as a consequence of upregulation of SOD2 and catalase upon osteogenic induction of human bone marrow-derived mesenchymal stem cells (BM-MSCs), while exogenous $\mathrm{H}_{2} \mathrm{O}_{2}$ retarded this osteogenic differentiation. Barbagallo et $\mathrm{al}^{42}$ revealed that increased expression of a major redox regulating gene, heme oxygenase-1, augmented osteogenesis in human BM-MSCs. Yamada et $\mathrm{al}^{16}$ showed that antioxidant $\mathrm{N}$-acetylcysteine was beneficial to osteogenesis of BM-MSCs and bone regeneration in rats. Particularly, Reid et $\mathrm{al}^{43}$ demonstrated that in human ADSCs, ROS were upregulated and downregulated during osteogenesis and adipogenesis, respectively. Therefore, we questioned if an antioxidant would be beneficial to osteogenic differentiation of human ADSCs. In the present work, we confirmed that osteogenic medium induced downregulation of ROS, and fullerol had an additive effect (Figure 2). Furthermore, either pretreatment or cotreatment of antioxidative fullerol promoted osteogenesis of human ADSCs in the presence of osteogenic induction (Figures 3 and 4). It is also demonstrated by an ongoing research project in our laboratory that fullerol has a similar impact on osteogenesis of mesenchymal stem cells from bone marrow. On the other hand, osteogenesis and adipogenesis are regarded to be closely correlated with the capacity of the progenitor cells during bone regeneration, and many reagents have been demonstrated to have opposite impacts on osteogenesis and adipogenesis. ${ }^{44-48}$ In this regard, we and others have recently reported that fullerol could inhibit the differentiation of marrow stromal cells and OP9 preadipocytes into adipocytes due to its scavenging activity against intracellular ROS. ${ }^{26,49,50}$ Taken together, these findings highlighted fullerol as a novel pharmaceutical candidate for the purpose of producing augmented bone regeneration. To this end, it is necessary to perform in vivo studies using fullerol-treated
ADSCs (for example, subcutaneous implantation) for bone regeneration.

FoxO1 belongs to the winged helix/forkhead family of transcription factors that have been shown to regulate expression of many genes involved in many cell processes, including cell proliferation, survival, differentiation, and redox balance. ${ }^{51}$ In particular, several recent studies revealed that FoxO1 is able to promote osteoblast differentiation of the progenitor cells..$^{20,21,52-54}$ However, its role in regulating osteogenic differentiation of ADSCs has not been reported yet. The data obtained from the present Western blot analysis showed that in human ADSCs, expression of FoxO1 was upregulated after addition of the osteogenic medium, and more importantly, cotreatment of fullerol with the osteogenic medium further augmented expression of this transcript factor and its significant targets Runx2, OCN, and SOD2 (Figure 5). Teixeira et $\mathrm{al}^{21}$ performed osteogenic induction using BMP-2 in mouse mesenchymal cells C3H10T1/2 and revealed that FoxO1 expression increased along with the osteogenic markers Runx2 and ALP during osteogenic differentiation. This is consistent with our present data (Figure 5) with regard to osteogenesis of human ADSCs in the presence of an induction medium containing AA, DEX, and GP, although Yang et $\mathrm{al}^{55}$ reported a negative role of FoxO1 in regulation of Runx2 and osteocalcin expression. Based on this preliminary data, we proposed a possible mechanism by which fullerol may exert its modulatory effect on osteoblast differentiation of mesenchymal stem cells through increasing FoxO1 and decreasing ROS (Figure 6). Additionally, SOD2

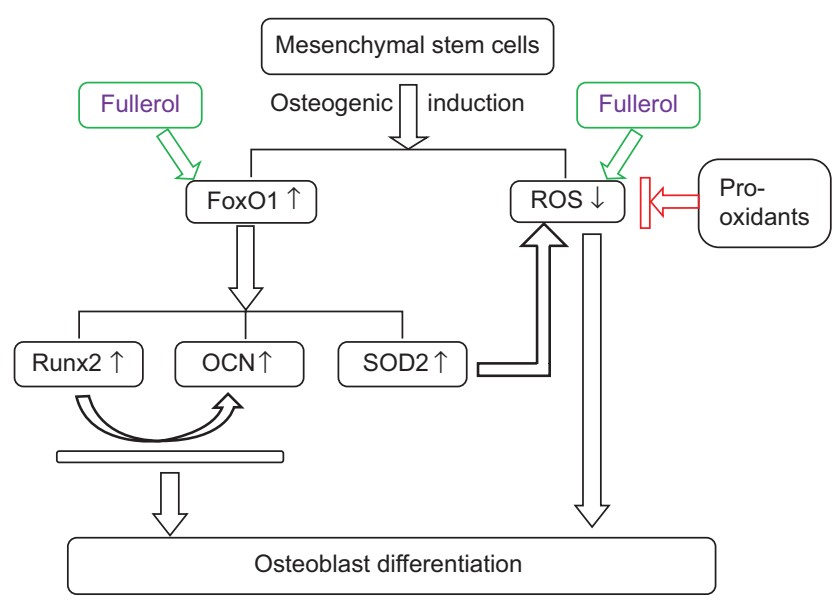

Figure 6 Schematic illustration of putative pathways for the enhancement of osteoblast differentiation of mesenchymal stem cells by fullerol.

Notes: Fullerol increases osteogenic markers Runx 2 and $\mathrm{OCN}$, and an antioxidative enzyme SOD2 through FoxOI. Meanwhile, fullerol supports osteoblast differentiation by the means of reducing the intracellular ROS level both directly and indirectly. Abbreviations: $\uparrow$, increase; $\downarrow$ decrease; OCN, osteocalcin; ROS, reactive oxygen species; Runx2, runt-related transcription factor 2; SOD2, superoxide dismutase 2. 
is hypothesized to play a role in diminishing ROS by fullerol, adding a new idea to the current ones on how fullerol exhibits its antioxidative activity within cells. ${ }^{56,57}$ Emphasis should be placed on the fact that the proposed mechanism is currently at its early stage and needs to be further verified by more detailed experimental evidence.

\section{Conclusion}

Our findings provided preliminary evidence that nanofullerols were able to enhance the osteogenesis of human ADSCs. This finding might offer potential opportunity for the use of nano-fullerol as alternatives to growth factors, serving as positive regulators of human ADSCs for the tissue engineering-based repair of bone fractures and defects.

\section{Acknowledgments}

The work was supported by the Orthopaedic Research and Education Foundation/Zachary B. Friedenberg Clinician Scientist Award and NIH/NIAMSR21 AR062732. The authors are thankful to Gina Beck of University of Virginia for her technical assistance in quantifying the Western blot images.

\section{Disclosure}

All authors have no conflict of interest to declare.

\section{References}

1. Haralson RH III, Zuckerman JD. Prevalence, health care expenditures, and orthopedic surgery workforce for musculoskeletal conditions. JAMA. 2009;302(14):1586-1587.

2. Aspenberg P, Genant HK, Johansson T, et al. Teriparatide for acceleration of fracture repair in humans: a prospective, randomized, doubleblind study of 102 postmenopausal women with distal radial fractures. J Bone Miner Res. 2010;25(2):404-414.

3. Szpalski C, Barbaro M, Sagebin F, Warren SM. Bone tissue engineering: current strategies and techniques - part II: Cell types. Tissue Eng Part B Rev. 2012;18(4):258-269.

4. Chen FH, Tuan RS. Mesenchymal stem cells in arthritic diseases. Arthritis Res Ther. 2008;10(5):223.

5. Casadei A, Epis R, Ferroni L, et al. Adipose tissue regeneration: a state of the art. J Biomed Biotechnol. 2012;2012:462543.

6. Cui L, Liu B, Liu G, et al. Repair of cranial bone defects with adipose derived stem cells and coral scaffold in a canine model. Biomaterials. 2007;28(36):5477-5486.

7. Tapp H, Hanley EN Jr, Patt JC, Gruber HE. Adipose-derived stem cells: characterization and current application in orthopaedic tissue repair. Exp Biol Med (Maywood). 2009;234(1):1-9.

8. Cheung WK, Working DM, Galuppo LD, Leach JK. Osteogenic comparison of expanded and uncultured adipose stromal cells. Cytotherapy. 2010;12(4):554-562.

9. Levi B, James AW, Nelson ER, et al. Human adipose derived stromal cells heal critical size mouse calvarial defects. PloS One. 2010;5(6):e11177.

10. James AW, Zara JN, Corselli M, et al. An abundant perivascular source of stem cells for bone tissue engineering. Stem Cells Transl Med. 2012; 1(9):673-684.

11. Levi B, James AW, Nelson ER, et al. Human adipose-derived stromal cells stimulate autogenous skeletal repair via paracrine Hedgehog signaling with calvarial osteoblasts. Stem Cells Dev. 2011;20(2): 243-257.
12. James AW, Zara JN, Zhang X, et al. Perivascular stem cells: a prospectively purified mesenchymal stem cell population for bone tissue engineering. Stem Cells Transl Med. 2012;1(6):510-519.

13. Zuk P, Chou YF, Mussano F, Benhaim P, Wu BM. Adipose-derived stem cells and BMP2: part 2. BMP2 may not influence the osteogenic fate of human adipose-derived stem cells. Connect Tissue Res. 2011; 52(2):119-132.

14. Cruz AC, Silva ML, Caon T, Simoes CM. Addition of bone morphogenetic protein type 2 to ascorbate and beta-glycerophosphate supplementation did not enhance osteogenic differentiation of human adipose-derived stem cells. J Appl Oral Sci. 2012;20(6): 628-635.

15. Chou YF, Zuk PA, Chang TL, Benhaim P, Wu BM. Adipose-derived stem cells and BMP2: part 1. BMP2-treated adipose-derived stem cells do not improve repair of segmental femoral defects. Connect Tissue Res. 2011;52(2):109-118.

16. Yamada M, Tsukimura N, Ikeda T, et al. N-acetyl cysteine as an osteogenesis-enhancing molecule for bone regeneration. Biomaterials. 2013;34(26):6147-6156.

17. Dugan LL, Gabrielsen JK, Yu SP, Lin TS, Choi DW. Buckminsterfullerenol free radical scavengers reduce excitotoxic and apoptotic death of cultured cortical neurons. Neurobiol Dis. 1996;3(2):129-135.

18. Mirkov SM, Djordjevic AN, Andric NL, et al. Nitric oxide-scavenging activity of polyhydroxylated fullerenol, $\mathrm{C} 60(\mathrm{OH}) 24$. Nitric Oxide. 2004; 11(2):201-207.

19. Xiao L, Aoshima H, Saitoh Y, Miwa N. Highly hydroxylated fullerene localizes at the cytoskeleton and inhibits oxidative stress in adipocytes and a subcutaneous adipose-tissue equivalent. Free Radic Biol Med. 2011;51(7):1376-1389.

20. Rached MT, Kode A, Xu L, et al. FoxO1 is a positive regulator of bone formation by favoring protein synthesis and resistance to oxidative stress in osteoblasts. Cell Metab. 2010;11(2):147-160.

21. Teixeira CC, Liu Y, Thant LM, Pang J, Palmer G, Alikhani M. FoxO1, a novel regulator of osteoblast differentiation and skeletogenesis. $J$ Biol Chem. 2010;285(40):31055-31065.

22. Zhang Y, Madhu V, Dighe AS, Irvine JN Jr, Cui Q. Osteogenic response of human adipose-derived stem cells to BMP-6, VEGF, and combined VEGF plus BMP-6 in vitro. Growth Factors. 2012;30(5): 333-343.

23. Livak KJ, Schmittgen TD. Analysis of relative gene expression data using real-time quantitative PCR and the 2(-Delta Delta C(T)) Method. Methods. 2001;25(4):402-408.

24. Kroto HW, Heath JR, O'Brien SC, Curl RF, Smalley RE. C-60 Buckminsterfullerene. Nature. 1985;318(6042):162-163.

25. Krusic PJ, Wasserman E, Keizer PN, Morton JR, Preston KF. Radical Reactions of C60. Science. 1991;254(5035):1183-1185.

26. Liu H, Yang X, Zhang Y, Dighe A, Li X, Cui Q. Fullerol antagonizes dexamethasone-induced oxidative stress and adipogenesis while enhancing osteogenesis in a cloned bone marrow mesenchymal stem cell. J Orthop Res. 2012;30(7):1051-1057.

27. Yang X, Ebrahimi A, Li J, Cui Q. Fullerene-biomolecule conjugates and their biomedicinal applications. Int J Nanomedicine. 2014;9: $77-92$.

28. Liu Q, Jin L, Mahon BH, Chordia MD, Shen FH, Li X. Novel treatment of neuroinflammation against low back pain by soluble fullerol nanoparticles. Spine (Phila Pa 1976). 2013;38(17):1443-1451.

29. Naim A, Shevlin PB. Reversible addition of hydroxide to the fullerenes. Tetrahedron Lett. 1992;33(47):7097-7100.

30. Li TB, Huang KX, Li XH, et al. Studies on the rapid preparation of fullerols and its formation mechanism. Chem J Chinese U. 1998;19(6): 858-860.

31. Injac R, Prijatelj M, Strukelj B. Fullerenol nanoparticles: toxicity and antioxidant activity. Methods Mol Biol. 2013;1028:75-100.

32. Bogdanovic G, Kojic V, Dordevic A, Canadanovic-Brunet J, VojinovicMiloradov M, Baltic VV. Modulating activity of fullerol $\mathrm{C} 60(\mathrm{OH}) 22$ on doxorubicin-induced cytotoxicity. Toxicol In Vitro. 2004;18(5): 629-637. 
33. Cai X, Jia H, Liu Z, et al. Polyhydroxylated fullerene derivative C(60) $(\mathrm{OH})(24)$ prevents mitochondrial dysfunction and oxidative damage in an MPP(+) -induced cellular model of Parkinson's disease. J Neurosci Res. 2008;86(16):3622-3634.

34. Chen YW, Hwang KC, Yen CC, Lai YL. Fullerene derivatives protect against oxidative stress in RAW 264.7 cells and ischemiareperfused lungs. Am J Physiol Regul Integr Comp Physiol. 2004; 287(1):R21-R26.

35. Srdjenovic B, Milic-Torres V, Grujic N, Stankov K, Djordjevic A, Vasovic V. Antioxidant properties of fullerenol $\mathrm{C} 60(\mathrm{OH}) 24$ in rat kidneys, testes, and lungs treated with doxorubicin. Toxicol Mech Methods. 2010;20(6):298-305.

36. Torres VM, Srdjenovic B, Jacevic V, Simic VD, Djordjevic A, Simplicio AL. Fullerenol C60 $(\mathrm{OH}) 24$ prevents doxorubicin-induced acute cardiotoxicity in rats. Pharmacol Rep. 2010;62(4):707-718.

37. Vapa I, Torres VM, Djordjevic A, et al. Effect of fullerenol C(60) $(\mathrm{OH})(24)$ on lipid peroxidation of kidneys, testes and lungs in rats treated with doxorubicine. Eur J Drug Metab Pharmacokinet 2012;37(4):301-307.

38. Xiao L, Takada H, Maeda K, Haramoto M, Miwa N. Antioxidant effects of water-soluble fullerene derivatives against ultraviolet ray or peroxylipid through their action of scavenging the reactive oxygen species in human skin keratinocytes. Biomed Pharmacother. 2005; 59(7):351-358.

39. Yudoh K, Shishido K, Murayama H, et al. Water-soluble C60 fullerene prevents degeneration of articular cartilage in Osteoarthritis via downregulation of chondrocyte catabolic activity and inhibition of cartilage degeneration during disease development. Arthritis Rheum. 2007; 56(10):3307-3318.

40. Mody N, Parhami F, Sarafian TA, Demer LL. Oxidative stress modulates osteoblastic differentiation of vascular and bone cells. Free Radic Biol Med. 2001;31(4):509-519.

41. Chen CT, Shih YR, Kuo TK, Lee OK, Wei YH. Coordinated changes of mitochondrial biogenesis and antioxidant enzymes during osteogenic differentiation of human mesenchymal stem cells. Stem Cells. 2008;26(4): 960-968.

42. Barbagallo I, Vanella A, Peterson SJ, et al. Overexpression of heme oxygenase-1 increases human osteoblast stem cell differentiation. J Bone Miner Metab. 2010;28(3):276-288.

43. Reid B, Afzal JM, McCartney AM, Abraham R, O’Rourke B, Elisseeff JH. Enhanced tissue production through redox control in stem cell-laden hydrogels. Tissue Eng Part A. 2013;19(17-18):2014-2023.

44. Park JG, Lee DH, Moon YS, Kim KH. Reversine increases the plasticity of lineage-committed preadipocytes to osteogenesis by inhibiting adipogenesis through induction of TGF-beta pathway in vitro. Biochem Biophys Res Commun. 2014;446(1):30-36.
45. Zhang L, Su P, Xu C, et al. Melatonin inhibits adipogenesis and enhances osteogenesis of human mesenchymal stem cells by suppressing PPARgamma expression and enhancing Runx 2 expression. J Pineal Res. 2010;49(4):364-372.

46. Li J, Zhang N, Huang X, et al. Dexamethasone shifts bone marrow stromal cells from osteoblasts to adipocytes by C/EBPalpha promoter methylation. Cell Death Dis. 2013;4:e832.

47. Nawa K, Ikeno H, Matsuhashi N, Ogasawara T, Otsuka E. Discovering small molecules that inhibit adipogenesis and promote osteoblastogenesis: unique screening and Oncostatin M-like activity. Differentiation. 2013;86(1-2):65-74.

48. Tseng PC, Hou SM, Chen RJ, et al. Resveratrol promotes osteogenesis of human mesenchymal stem cells by upregulating Runx 2 gene expression via the SIRT1/FOXO3A axis. J Bone Miner Res. 2011;26(10): 2552-2563.

49. Saitoh Y, Xiao L, Mizuno H, et al. Novel polyhydroxylated fullerene suppresses intracellular oxidative stress together with repression of intracellular lipid accumulation during the differentiation of OP9 preadipocytes into adipocytes. Free Radic Res. 2010;44(9):1072-1081.

50. Liu Q, Jin L, Shen FH, Balian G, Li XJ. Fullerol nanoparticles suppress inflammatory response and adipogenesis of vertebral bone marrow stromal cells - a potential novel treatment for intervertebral disc degeneration. Spine J. 2013;13(11):1571-1580.

51. Kousteni S. FoxO1: a molecule for all seasons. J Bone Miner Res. 2011; 26(5):912-917.

52. Kim KM, Park SJ, Jung SH, et al. miR-182 is a negative regulator of osteoblast proliferation, differentiation, and skeletogenesis through targeting FoxO1. J Bone Miner Res. 2012;27(8):1669-1679.

53. Moriishi T, Kawai Y, Komori H, et al. Bcl2 deficiency activates FoxO through Akt inactivation and accelerates osteoblast differentiation. PloS One. 2014;9(1):e86629.

54. Siqueira MF, Flowers S, Bhattacharya R, et al. FoxO1 modulates osteoblast differentiation. Bone. 2011;48(5):1043-1051.

55. Yang $\mathrm{S}, \mathrm{Xu} \mathrm{H}, \mathrm{Yu} \mathrm{S}$, et al. FoxO1 mediates insulin-like growth factor 1 (IGF1)/insulin regulation of osteocalcin expression by antagonizing Runx2 in osteoblasts. J Biol Chem. 2011;286(21):19149-19158.

56. Satoh M, Takayanagi I. Pharmacological studies on fullerene (C60), a novel carbon allotrope, and its derivatives. J Pharmacol Sci. 2006; 100(5):513-518.

57. Chistyakov VA, Smirnova YO, Prazdnova EV, Soldatov AV. Possible mechanisms of fullerene $\mathrm{C}(6)(0)$ antioxidant action. Biomed Res Int. 2013;2013:821498.
International Journal of Nanomedicine

\section{Publish your work in this journal}

The International Journal of Nanomedicine is an international, peerreviewed journal focusing on the application of nanotechnology in diagnostics, therapeutics, and drug delivery systems throughout the biomedical field. This journal is indexed on PubMed Central, MedLine, CAS, SciSearch $®$, Current Contents $\AA /$ Clinical Medicine,

\section{Dovepress}

Journal Citation Reports/Science Edition, EMBase, Scopus and the Elsevier Bibliographic databases. The manuscript management system is completely online and includes a very quick and fair peer-review system, which is all easy to use. Visit http://www.dovepress.com/ testimonials.php to read real quotes from published authors. 